\title{
Design and testing of a low impedance transceiver circuit for nitrogen-14 nuclear quadrupole resonance
}

Hideo Sato-Akaba

Department of Systems Innovation, Graduate School of Engineering Science, Osaka

University, Toyonaka, Osaka 992-8510, Japan

Author to whom correspondence should be addressed:

Hideo Akaba, Ph.D.

Department of Systems Innovation, Graduate School of Engineering Science,

Osaka University

Toyonaka, Osaka 992-8510, Japan

Phone: +81-6-6850-6313

Fax: +81-6-6850-6312

E-mail : akaba@sup.ee.es.osaka-u.ac.jp

Keywords: nitrogen-14 nuclear quadrupole resonance; transimit-reciever switch circuit; class-D amplifier; transimpedance amplifier; Q switch; non-invasive detection; illicit drugs; explosives; counterfeit medicines

\begin{abstract}
A low impedance transceiver circuit consisting of a transimit-recieve switch circuit, a class-D amplifier and a transimpedance amplifier (TIA) was newly designed and tested for a nitrogen-14 NQR. An NQR signal at $1.37 \mathrm{MHz}$ from imidazole was successfully observed with the dead time of $\sim 85 \mu \mathrm{s}$ under the high $\mathrm{Q}$ transmission $(\mathrm{Q} \sim 120)$ and reception $(\mathrm{Q} \sim 140)$. The noise performance of the low impedance TIA with an NQR probe was comparable with a commercial low noise $50 \Omega$ amplifier (voltage input noise: $0.25 \mathrm{nV} / \sqrt{\mathrm{Hz}}$ ) which was also connected to the probe. The protection voltage for the pre-amplifier using the low impedance transceiver was $\sim 10$ times smaller than that for the pre-amplifier using a $50 \Omega$ conventional transceiver, which is suitable for NQR remote sensing applications.
\end{abstract}




\section{Introduction}

Nuclear quadrupole resonance (NQR) is similar to nuclear magnetic resonance (NMR) in which energy gaps are generated by an external static magnetic field. Nuclei with a spin of one or more have electric quadrupole moments, and therefore their energies are split by the intrinsic electric field gradient, which is highly specific to the substance. Therefore, an additional external static field is not necessary to generate the energy gaps for NQR. One of the promising applications using nitorgen-14 nuclear quadrupole resonance (NQR) is non-invasive detection such as explosive substances [1-7], illicit drugs [8-11] and counterfeit medicines [12-15]. Especially in Japan, the non-invasive detection of methamphetamine hydrochloride to prevent from smuggling is expected by Japan Customs since it has occupied $80 \%$ of all detected illicit drugs in each year. The NQR frequencies from methamphetamine hydrochloride were recently confirmed at $1.217 \mathrm{MHz}$ and $0.654 \mathrm{MHz}$ [11], which is in the frequency range $(0.5 \mathrm{MHz} \sim 5 \mathrm{MHz})$ for ${ }^{14} \mathrm{~N} \mathrm{NQR}$. The phase memory time $T_{2}{ }^{*}(\sim 0.7 \mathrm{~ms}$ for $1.217 \mathrm{MHz})$ is relatively short in this frequency range so that shortening dead time of the receiver is important [11].

However, the detection hardware consisting of a commercial NQR/NMR console, a power amplifier and a pre-amplifier becomes expensive for such applications. To lower the cost of the construction, a low impedance transceiver circuit was newly designed and tested using a mobile NQR/NMR console in this work. So far a low impedance series resonant circuit using a switching amplifier such as class-D/E amplifier has been used for generation of an RF magnetic field [16-19]. Some of works have been mentioned about the high impedance reception using a high impedance amplifier $[18,19]$. In this case, a protection circuit for the high impedance amplifier becomes an issue for NQR remote sensing applications where a high voltage over several $\mathrm{kV}$ is generating across an excitation/detection coil. To lower the protection voltage, low impedance NMR/NQR reception $(0.5 \sim 5 \mathrm{MHz})$ combined with the low impedance NMR/NQR transmission $(0.5 \sim 5 \mathrm{MHz})$ was proposed. In addition, to lower the dead time of the reception a class-D amplifier with small modifications to change the internal resistance of the amplifier at the falling edge of the transmission was developed.

For demonstration of the low impedance transmission and reception, we measured ${ }^{14} \mathrm{~N} N Q R$ from imidazole which NQR frequency $(1.368 \mathrm{MHz}$ at $300 \mathrm{~K})$ is similar to that from methamphetamine hydrochloride.

\section{Methods}

\subsection{NQR spectrometer}

All NQR experiments were performed on a homemade FPGA (field-programmable gate 
array) based NQR/NMR console with a proposed transceiver circuit. The console was constructed mainly from an FPGA developing board (DE0-NANO, Terasic Technologies Inc.), an FT2232H Mini-Module (Future Technology Devices International Ltd), a receiver amplifier made from an operational amplifier IC (LT6201, Linear Technology Corp.), an analog digital (A/D) converter (LTC2356-14) and a band pass filter (LT1568). The spectrometer was controlled using a laptop PC (MacBook Air, Apple Inc.) via USB connection. The FPGA hardware program was developed with Quartus II v10 (Altera Corp.). The NQR control software was written by LabVIEW 2012 (National Instruments Corp.) and Xcode 4.2.1 (Apple Inc.).

An output signal from a pre-amplifier was fed into the receiver amplifier and then filtered with the band pass filter before digitizing the signal with the A/D converter. The output of the serial signal from the A/D converter connected to the general purpose input/output (GPIO) in the FPGA board. The sampling frequency was selected as NQR frequency $\times 4$ divided by odd number for digital quadrature detection using band pass sampling [20-23]. Two PWM signals were out from the GPIO in the FPGA board to drive a class-D amplifier. The phase of the PWM1 signal was 180 degree different from that of the PWM2. A gate signal to switch the Q factor of the receiver circuit between the low and high Q state was also out from the GPIO in the FPGA board.

\subsection{Transmit-receive switch circuit}

One of the advantages for using a series resonant circuit as shown in Fig. 1 (a) is that a large current from a low voltage source at the resonant frequency is possible since the resistance of the circuit can be less than a few $\Omega$ mainly owing to an inductor coil and leads between the electronic parts. Therefore, a large magnetic field from the coil is generated with a low voltage power amplifier for the transmission. If the resistance of the circuit becomes smaller, the quality (Q) factor of the circuit increases and then voltage amplification becomes larger. On the other hand, the reception of NQR/NMR signal using the series resonant circuit has a disadvantage that a general $50 \Omega$ low noise amplifier (LNA) is not suitable since the loaded $Q$ factor of the resonant circuit becomes too small. Generally, a parallel capacitor $\mathrm{Cp}$ to the coil is inserted for the impedance matching as shown in Fig. 1 (b). In this case, the Q factor of the resonant circuit becomes half of the unloaded $\mathrm{Q}$ factor. To prevent the decrease of the $\mathrm{Q}$ factor, a transimpedance amplifier (TIA) with low input impedance was proposed in this work. To optimize the noise performance of the TIA inserted in the resonant circuit, a parallel capacitor $\mathrm{Cp}$ to the coil was inserted as shown in Fig. 1 (b).

To combine the transmitter and receiver circuits as simple as possible, a transmit-receive 
switch circuit as shown in Fig. 1 (c) was newly designed. An NQR probe consisted of fixed capacitors (C Series: high voltage, TDK Corp.), a variable capacitor (NMTM120C, Voltronics Corp.) and a solenoid coil ( $\phi 44 \mathrm{~mm} \times 70 \mathrm{~mm}, \mathrm{~L}=29.1 \mu \mathrm{H}, \mathrm{R}=1.53 \Omega$ at $1.37 \mathrm{MHz})$. The precise resonant frequency for the transmission and reception was tuned with changing the value of Cs2", where Cs2" consisted of fixed capacitors and a variable capacitor. During the transmission, this circuit is simplified to be a series resonant circuit with the high quality (Q) factor as shown in Fig. 1 (a) since diodes of D1 D4 (SBYV27-200, Vishay General Semiconductor) become low impedance with the applied voltage more than the forward voltage drop of the diode, and T/R switch ICs of TR1, TR2 (MD0100, Supertex Inc.) and TR3 (MD0105) become high impedance with the applied voltage exceeding a value of $\pm 2.0 \mathrm{~V}$. In contrast, during the reception, the circuit becomes equivalent to a resonant circuit consisting of series and parallel capacitors, and the inductor coil as shown in Fig. 1 (b) since diodes of D1 D4 become high impedance and T/R switch ICs of TR1, TR2 and TR3 become low impedance. The on resistance of MD0100 is typically $15 \Omega$, while that of MD0105 can be $\sim 3$ $\Omega$ when the four channels in the IC package are connected together in parallel. In addition, the impedance matching to $50 \Omega$ was also possible even if a general $50 \Omega$ pre-amplifier was used in the receiver circuit.

The maximum protection voltage for the pre-amplifier was supposed to be $\pm 110 \mathrm{~V}$ due to the specification of the T/R switch IC (MD0100). A bipolar transistor Q1 (FZT853, Diodes Inc.) was used to change the $\mathrm{Q}$ factor of the receiver circuit by switching the resistance of the receiver circuit from $\sim 25 \Omega$ to $\sim 1.6 \Omega$ that was controlled by the gate signal generated from the NQR/NMR console. A capacitor $\mathrm{C} 1$ and a resistor R1 worked as a low pass filter to suppress the quick response of this switch action intending to minimize a ringing signal caused by switching of the transistor Q1.

\subsection{Receiver pre-amplifier}

A resistive feedback transimpedance amplifier (TIA) with low input impedance was adopted using a single low-noise and high-speed operational amplifier (op-amp) (CLC1001, CADEKA Microcircuits LLC. or LT6200-10, Linear Technology Corp.). The configuration is a basic inverted amplifier with a single voltage supply as low as $4 \mathrm{~V}$. The circuit diagram is shown in Fig. 2. This amplifier became a part of the series resonant circuit during the reception. Virtual short of the op-amp with a feed back circuit makes the input impedance low (ideally zero). The capacitor C2 was necessary to block a DC current to the protection diodes (1N4148, Fairchild Semiconductor) generating from an input DC bias voltage of the op-amp. 
In addition, it was used to adjust the small difference of the resonance frequency between the receiver circuit and transmission circuit. The noise properties of the op-amps from the manufacturer's datasheets were listed in Table.1.

\subsection{Noise simulation and measurement for the receiver pre-amplifier}

Output spectral noise densities of the pre-amplifiers connected with the probe were simulated with LTspice IV for OSX (Linear Technology Corp.) and experimentally measured with a vector signal analyzer (8941A, Agilent Technologies Inc.). The probe consisted of a solenoid coil $(29.1 \mu \mathrm{H}$ and $1.53 \Omega$ at $1.37 \mathrm{MHz})$ and series (Cs and Cs") and parallel (Cp) capacitors as shown in Fig. 1 (b). The probe and pre-amplifier was in the metal shield box. A battery power supply ( $5 \mathrm{~V}$ ) was used to minimize the noise from the voltage supply. The input impedance of the vector signal analyzer was set at $50 \Omega$ that become a load for the amplifier. For the simulation, the additional resistance was inserted owing to the resistance from the leads and the input impedance of the pre-amplifier. For optimization of the noise performance, the values of $\mathrm{Cs}$ and $\mathrm{Cp}$ were varied with the condition that the value of $\mathrm{Cp}+$ Cs was kept constant in order to keep the resonant frequency similar.

To compare with three commercial wideband low noise pre-amplifiers (LNAs) with $50 \Omega$ input impedance, which are listed in Table.1, a reference signal radiated from a single loop coil $(\phi 20 \mathrm{~mm})$ was used since the gain of the amplifiers and the $\mathrm{Q}$ factor of the resonant circuit became different. The loop coil was fixed near the one side of the probe coil with the distance of $10 \mathrm{~mm}$. The reference signal fed to the coil was generated from a function generator (WF1946B, NF Corp.) and the output voltage was set at $10 \mathrm{mV}$. Impedance matching was conducted by a network analyzer (MS2024B, Anritsu Corp.) for the $50 \Omega$ reception system.

\subsection{Transmitter power amplifier}

A class-D amplifier with low output impedance $(<1 \Omega)$ and a $Q$ switch function was designed with a half bridge configuration. The circuit diagram is shown in Fig. 3. This amplifier became a part of the series resonant circuit during the transmission. Therefore, a rise and fall time of the current through the circuit depends on the $\mathrm{Q}$ factor of the circuit.

For the Q switch function, diodes D6, D7 (SBYV27-200), D8 (1N4760A, Fairchild Semiconductor Corp.), and a resistance R4 (25 $\Omega$ ) were inserted. Diodes D5, D8 were used for clipping the voltage less than the breakdown voltage of MOSFETs Q2 and Q3 (STP16NF06, STMicroelectronics) and the TR switch IC TR4. Two PWM signals (PWM1 and PWM2) were fed into a MOSFET gate driver (LM5101a, Texas Instruments Inc.). The 
phase of the PWM2 signal was 180 degree different from that of the PWM1. During the transmission, the output impedance of the amplifier was less than $1 \Omega$, which was mainly from the on resistance of the MOSFETs. After the transmission, both MOSFETs were in the off state. However, the transmission signal still remained in the resonant circuit and decayed exponentially with time. Before the signal voltage less than a forward voltage drop of the diodes D6 and D7, the output impedance of the amplifier was dominated by the value of the resister $\mathrm{R} 4=25 \Omega$, which reduced the $\mathrm{Q}$ factor of the circuit.

The shape of the transmission signal was captured with the same loop coil that was used for sending the reference signal to the probe during the reception, although the output of the loop coil directly connected to the receiver amplifier of the NQR/NMR console. For measurement of the voltage across the probe coil, two 100 $\times$ scope probes were inserted between one of the terminals of the probe coil and the ground. The voltage across the coil was calculated by subtraction of the voltages observed at the both terminals of the coil.

\subsection{Estimation of the $Q$ factor}

The involved components for the resonant circuits were different between the transmission and reception duration, which resulted in the different $\mathrm{Q}$ factor of the circuit between the transmission and reception duration. For the transmission, the Q factor was estimated from $\pi f \tau$ where $f$ is a resonance frequency and $\tau$ is an exponential time constant of the rising or falling edges of the transmission signal. For the reception, the $3 \mathrm{~dB}$ bandwidth of the reception system was measured with the vector signal analyzer by sweeping a frequency of the reference signal radiated from the single loop coil. The $\mathrm{Q}$ factor was estimated by $f_{c} /$ $f_{b w}$ where $f_{c}$ is a resonant frequency and $f_{b w}$ is a bandwidth of the resonant circuit.

\subsection{NQR measurement}

To verify that the proposed low impedance transceiver circuit could acquire NQR signals, we measured ${ }^{14} \mathrm{~N}$ NQR free induction decay (FID) signals from imidazole $\left(\mathrm{C}_{3} \mathrm{H}_{4} \mathrm{~N}_{2}\right)$ with one pulse sequence at room temperature. A plastic tube containing $30 \mathrm{~g}$ powered sample was inserted into the probe coil $(\phi 44 \mathrm{~mm} \times 70 \mathrm{~mm}, \mathrm{~L}=29.1 \mu \mathrm{H}, \mathrm{R}=1.53 \Omega$ at $1.37 \mathrm{MHz})$. The probe coil and transmit-receive switch circuit except the power amplifier were shielded with an aluminum metal box separately from the NQR console. The values of capacitor Cs2, Cs2" and $\mathrm{Cp}$ were $141 \mathrm{pF}, 821 \mathrm{pF}$ and $900 \mathrm{pF}$, respectively. The parameters for the NQR acquisition were as follows: RF frequency for excitation $1.370 \mathrm{MHz}, \mathrm{RF}$ reference frequency $1.374 \mathrm{MHz}$, VDD (a power supply voltage) for the class-D amplifier amplifier $12 \mathrm{~V}$, pulse width $100 \mu \mathrm{s}$, repetition time $3.0 \mathrm{~s}$ and number of scan 300 . 


\section{Results and discussion}

\subsection{Noise performance of the receiver pre-amplifier}

The measured and simulated output spectral noise densities from the transimpedance pre-amplifiers (TIAs) connected with the probe are shown in Fig. 4. To fit the measured spectrum for CLC1001, the parameters for the op-amp were fixed as follows: the input voltage noise was $0.55 \mathrm{nV} / \sqrt{\mathrm{Hz}}$ and the input current noise was $4.25 \mathrm{pA} / \sqrt{\mathrm{Hz}}$. In the case of LT6200-10, the input voltage and current noise was $0.9 \mathrm{nV} / \sqrt{\mathrm{Hz}}$ and $3.37 \mathrm{pA} / \sqrt{\mathrm{Hz}}$, respectively. Small discrepancy of the input voltage and current noises from the datasheet was necessary for the fittings of the measurement data. The variable parameters were resistance of the circuit to adjust bandwidth and amplitude of the noise spectrum and capacitor Cs" to adjust small difference of the resonant frequency. The values obtained from the fittings were summarized in the Table 2.

The solid lines indicating a simulated total noise were matched with the measured data well, while the dotted lines $n_{r}$ and $n_{\text {amp }}$ were spectral noise densities contributed from resistance of the resonant circuit and from the TIA, respectively. The noise figures (NFs) at the resonant frequency were also calculated from the noise contributions as the following equation: $\mathrm{NF}=$ $20 \log 10\left(V_{\text {total }} / V_{\mathrm{r}}\right)$, where $V_{\text {total }}$ is the output total noise density from the TIA and $V_{\mathrm{r}}$ is the thermal noise contribution from the resistor included in $V_{\text {total }}$. These results indicate that the series resonant circuit for the receiver was not preferable from the noise point of view.

As the Cs became larger which means the impedance of the probe decreased, the amplifier noise was more dominant in the total noise around the resonant frequency. This is due to the increase of the current noise converted from the input voltage noise of the op-amp by the smaller impedance of the probe. This current noise is further converted to the output voltage noise by the feed back resistor R3 (Fig. 2). Meanwhile, the contribution from the input current noise of the op-amp is independent from the value of $\mathrm{Cs}$ and $\mathrm{Cp}$. As the Cs became smaller, the input current noise of the amplifier was more dominant in the total noise. This is due to the decrease of the current noise converted from the input voltage noise of the op-amp by the larger impedance of the probe. Therefore, the optimized point for $\mathrm{Cs}$ and $\mathrm{Cp}$ depends on the noise properties of the op-amp. The signal to noise ratio (SNR) of the two TIAs plotted as a function of Cs is shown in Fig. 5, where SNR was defined by $V_{\text {ref }} / V_{\text {total }}$ measured at the resonant frequency. The optimum points for LT6200-10 and CLC1001 were different due to the different noise properties of the op-amp.

The peak of the spectral noise density was due to the resonance of the circuit since the current generated from the parasitic resistor in the coil depended on the probe impedance. 
Therefore, the contribution of the resistance noise was minimizing outside of the resonant frequency. This is useful to find an exact resonant frequency of the circuit by measuring the noise spectrum.

The resistance obtained from the fitting was similar to the measured resistance of the probe coil for CLC1001 and LT6200-10. However the small discrepancies appeared when the Cs became large. These discrepancies of 1 2 $\Omega$ should be caused by the input impedance of the op-amp.

The SNRs from three commercial LNAs with $50 \Omega$ input impedance were compared with the proposed TIA as shown in Fig. 4 (d). Because of the different gains of the amplifiers, the amplitudes of the spectral noise densities for the $50 \Omega$ system were normalized so that reference signal intensities become same among them. The SNR of the TIA using CLC1001 was comparable with the amp3 with the input voltage noise as low as $0.25 \mathrm{nV} / \sqrt{\mathrm{Hz}}$.

\subsection{Transmitter power amplifier}

Envelopes of the excitation pulse signals were plotted as a function of time for three configurations as shown in Fig. 6. The signal for the config. 1 was obtained under the following condition; diodes D6, D7 in the class-D amplifier (Fig. 3), D1, D4 in the transmit-receive switch circuit (Fig.1 (c)) were bypassed by wirings and the low side MOSEFT Q3 (Fig. 3) was kept in the on state during the falling time of the transmission. The Q factor was 113 and 160 estimated from the rising and falling edge, respectively. The signal for the config. 2 was obtained with no change from the proposed circuit except the change of the low side MOSEFT Q3 setting at the on state during the falling time of the transmission. The Q factor was 115 estimated from the rising edge, and was gradually decreased from 100 to 10 from the falling edge. The decrease of the $\mathrm{Q}$ factor was due to the resistance across the diode that is dependent on the applied voltage. The signal for the config. 3 was obtained with no change from the proposed circuit. The Q factor was 117 from the rising edge, and was gradually decreased from 15 to 10 during the falling edge.

From the results, resistance change of the diodes depending on the applied voltage made decay time constant shorter. Moreover, setting the low side MOSEFT at the off state after the end of the transmission additionally lowered the decay time, which was caused by the resistance of the $\mathrm{R} 4=25 \Omega$. Figure 7 shows the voltage across the coil of the probe as a function of VDD that was a drain voltage of the upper side MOSFET Q2. The systematic offsets between the config. 1 and the config. 3 were due to the forward voltage drop of the diodes. Therefore, a diode with a low voltage forward drop is suitable as well as a small junction capacitance. For comparison with the $50 \Omega$ transmission system, the coil voltage 
plotted as a function of the output voltage from the $50 \Omega$ power amplifier (N146-6034A, Thamway Co. Ltd.) is also shown in Fig.7. The voltage amplification factor was 10 times smaller than the low impedance transmission system. In other word, the protection voltage for the electronic parts for the low impedance system could be 10 times smaller than the $50 \Omega$ transmission system.

\subsection{Transmit-receive switch circuit}

An NQR signal from imidazole, which was resolved into quadrature components by bandpass sampling, was successfully observed with the proposed transmit-receive switch circuit as shown in Fig.8. The envelope of the excitation pulse and the Q factor of the circuit are indicated in the upper figures. The pulse-like signal appeared at $\sim 465 \mu$ s was leak from the rising edge of the gate signal to the receiver amplifier in the NQR console and indicated the Q switch timing. In Fig. 8 (a), the signal intensity at the high Q (140) state during the reception was $\sim 10$ times larger than the intensity at the low Q (13) state, which was consistent with the ratio of the high $\mathrm{Q}$ and low $\mathrm{Q}$ factor.

The early part of the received signal, which was saturated with the excitation pulse due to leakage to the pre-amplifier, was deleted from the plot. The dead time $t_{\text {dead }}$ of the receiver was confirmed around $85 \mu$ s when the applied voltage was up to $3 \mathrm{kVpp}$ across the coil, and $t_{\text {delay }}$ was optimized at $50 \mu \mathrm{s}$ as shown in Fig. 8(b). When the Q factor of the receiver circuit was changed immediately at the end of the excitation pulse, the dead time was $\sim 500 \mu$ s (data not shown). Therefore, reduction of the dead time using this transmit-receive switch circuit was verified.

\subsection{The cost for the construction}

The cost of the construction is important factor for the real applications such as inspections of illegal drugs, counterfeit medicines and medicine qualification. The total cost of the electronic parts for the designed low impedance transceiver circuits was less than US\$100 except the variable capacitor used in the probe, which is considerably lower cost compared with general $50 \Omega$ conventional wideband transceivers. In addition, all of the electronic parts used in the circuits were commercially available.

\section{Conclusions}

A low impedance transceiver circuit for a nitrogen-14 NQR was newly designed and tested. High Q transmission $(\mathrm{Q} \sim 120)$ and reception $(\mathrm{Q} \sim 140)$ were successfully done. During the transmission, the circuit became a series resonant circuit. The supply voltage of $20 \mathrm{~V}$ for the 
class-D amplifier was amplified up to $2.5 \mathrm{kVpp}$ across the coil. During the reception, an additional capacitor parallel to an excitation/detection coil was inserted to increase signal to noise ratio. The designed transimpedance amplifier (TIA) mainly consisted of a low noise single operational amplifier. The input impedance of the TIA was less than $2 \Omega$. Noise performance of the TIA was comparable with that of low noise $50 \Omega$ amplifies. To decrease a dead time of the reception to less than $100 \mu \mathrm{s}$, the $\mathrm{Q}$ factor of the transceiver circuit was switched to 13 between the end of the transmission and before the reception. The protection voltage for the pre-amplifier using the low impedance transceiver was $\sim 10$ times smaller than that for the pre-amplifier using a $50 \Omega$ conventional transceiver, which is suitable for NQR remote sensing applications.

\section{Acknowledgements}

The author would like to thank Dr. H. Itozaki and Dr. Y. Miyato (Osaka Univeristy) for useful advice during the course of this work. This work was supported by Japan Science and Technology Agency (JST) A-STEP program.

\section{Reference}

[1] V.S. Grechishkin, N.Y. Sinyavskii, New technologies: nuclear quadrupole resonance as an explosive and narcotic detection technique, Phys. Usp. 40 (1997) 393-406.

[2] A.N. Garroway, M.L. Buess, J.B. Miller, B.H. Suits, A.D. Hibbs, G.A. Barrall, R. Matthews, L.J. Burnett, Remote sensing by nuclear quadrupole resonance, IEEE T. Geosci. Remote 39 (2001) 1108-1118.

[3] R.M. Deas, I.A. Burch, D.M. Port, Detection of RDX and TNT mine-like targets by nuclear quadrupole resonance, Proc. SPIE 4742 (2002) 482-489.

[4] J. Barras, M.J. Gaskell,N. Hunt, R.I. Jenkinson,K.R. Mann, D.A.G. Pedder, G.N. Shilstone, J.A.S Smith, Detection of ammonium nitrate inside vehicles by nuclear quadrupole resonance, Appl. Magn. Reson. 25(3-4) (2004) 411-437

[5] A. Gregorovic, T. Apih, TNT detection with 14N NQR: Multipulse sequences and matched filter, J. Magn. Reson. 198 (2009) 215-221.

[6]M. Ostafin, B. Nogaj,N-14-NQR based device for detection of explosives in landmines, MEASUREMENT, 40(1) (2007) 43-54.

[7] J.A.S. Smith, T.J. Rayner, M.D. Rowe, J. Barras, N.F. Peirson, A.D. Stevens, K. Althoefer, Magnetic field-cycling NMR and (14)N, (17)O quadrupole resonance in the explosive pentaerythritol tetranitrate (PETN), J. Magn. Reson. 204 (2010) 139-144. 
[8] J.P. Yesinowski, M.L. Buess, A.N. Garroway, M. Ziegeweid, A. Pines, Detection of ${ }^{\mathrm{I4}} \mathrm{N}$ and ${ }^{35} \mathrm{Cl}$ in Cocaine Base and Hydrochloride Using NQR, NMR, and SQUID Techniques, Anal. Chem. 67 (1995) 2256-2263.

[9] T.N. Rudakov, P.A. Hayes, J.H. Flexman, Optimised NQR pulse technique for the effective detection of heroin base, Solid State Nucl. Magn. Reson. 33 (2008) 31-35.

[10] J. Shinohara, K. Kobayashi, H. Sato-Akaba, H. Itozaki, Nuclear quadrupole resonance of norephedrine, Solid State Nucl. Magn. Reson. 40(3) (2011) 121-125.

[11] J. Shinohara, H. Sato-Akaba, H. Itozaki, Nuclear quadrupole resonance of methamphetamine hydrochloride, Solid State Nucl. Magn. Reson. 43-44 (2012) 27-31.

[12] E. Balchin, D.J. Malcolme-Lawes, L.J.F. Poplett, M.D. Rowe, J.A.S. Smith, G.E.S. Pearce, S.A.C. Wren, Potential of nuclear quadrupole resonance in pharmaceutical analysis, Anal. Chem. 77 (2005) 3925-3930.

[13] J. Barras, S. Katsura, H. Sato-Akaba, H. Itozaki, G. Kyriakidou, M.D. Rowe, K.A. Althoefer, J.A.S. Smith, Variable-Pitch Rectangular Cross-section Radiofrequency Coils for the Nitrogen-14 Nuclear Quadrupole Resonance Investigation of Sealed Medicines Packets, Anal. Chem., 84 (21) (2012) 8970-8972.

[14] J. Barras, D. Murnane, K. Althoefer, S. Assi, M. D. Rowe, I. J. F. Poplett, G. Kyriakidou, and J. A. S. Smith, Nitrogen-14 Nuclear Quadrupole Resonance Spectroscopy: A Promising Analytical Methodology for Medicines Authentication and Counterfeit Antimalarial Analysis, Anal. Chem. 85 (5) (2013) 2746-2753.

[15] J. Luznik, J. Pirnat,V. Jazbinsek, Z. Lavric, S. Srcic, Z. Trontelj, The Influence of Pressure in Paracetamol Tablet Compaction on N-14 Nuclear Quadrupole Resonance Signal, Appl. Magn. Reson., 44(6) 2013 735-743.

[16] R. A. Slade, RF magnetic field pulse generator, U.S. Patent 6002256 A Dec, 14, 1997.

[17] Cherik Bulkes, Stephen Denker, Arthur J. Beutler, Magnetic resonance imaging system with a Class-E radio frequency amplifier, U.S. Patent 7382128 B2, June, 3, 2008.

[18] N. Sun, T. J. Yoon, H. Lee, W. Andress, R. Weissleder, and D. Ham, Palm NMR and one-chip NMR, IEEE J. Solid-St. Circ. 46 (2011) 342-352.

[19] X. Zhang, N. Schemm, S. Balkir, M.W. Hoffman, A Low-Power Compact NQR Based Explosive Detection System, IEEE Sensors J. 14 (2) (2014) 4497-507

[20] R. G. Vaughan, N. L. Scott, D. R. White, The theory of bandpass sampling, IEEE Trans. Signal Process. 39(9) (1991) 1973-1984.

[21] P. Perez, J. J. Vaquero, A. Santos, M. Rivera, F. del Pozo, Applying undersampling to the nuclear magnetic resonance signal, Proc. IEEE EMBS 2 (1996) 716-717.

[22] J.S. Hyde, H.S. McHaourab, T.G. Camenisch, J.J. Ratke, R.W. Cox, W. Froncisz 
Electron paramagnetic resonance detection by time-locked subsampling, Rev. Sci. Instrum. 69 (1998), 2622-2628.

[23] G. Giovannetti, V. Hartwig, V. Viti, G. Gaeta, R. Francesconi, L. Landini, A. Benassi, Application of undersampling technique for the design of an NMR signals digital receiver, Concept Magn. Reson. B 29B (3) (2006) 107-114. 


\section{Figure caption}

Fig. 1 A series resonant circuit for the transmission (a) and a resonant circuit with series and parallel capacitors to a inductor coil for the reception (b). A proposed transmit-receive switch circuit combined with a resonant circuit (c).

Fig. 2 A designed transimpedance amplifier made from a single op-amp. As a low noise op-amp U1 in the pre-amplifier, each of CLC1001 and LT6200-10 was evaluated.

Fig. 3 A proposed half bridge class-D amplifier with a $\mathrm{Q}$ switch function. The resistor $\mathrm{R} 4$ determines the $\mathrm{Q}$ factor of the series resonant circuit during a falling edge of the transmission signal. Two PWM signals fed into the MOSFET gate driver are shown in the figure.

Fig. 4 Spectral noise densities of the TIA made from CLC1001 connected with the NQR probe with following conditions: (a) $\mathrm{Cs}=20 \mathrm{pF}, \mathrm{Cp}=1021 \mathrm{pF}$, (b) $\mathrm{Cs}=141 \mathrm{pF}, \mathrm{Cp}=900 \mathrm{pF}$ (c) $\mathrm{Cs}=1041 \mathrm{pF}, \mathrm{Cp}=0.0$ $\mathrm{pF}$ in the probe. The $n_{\mathrm{r}}$ was a simulated noise contribution from the resistor in the probe, while $n_{\text {amp }}$ was the contribution from the TIA itself. The solid lines in (a-c) indicate the total simulated noise densities. (d) The noise densities of three commercial $50 \Omega$ LNAs, compared with that of the TIA (CLC1001).

Fig. 5 SNRs of the TIAs (OCLC1001, $\triangle$ LT6200-10) with the NQR probe plotted as a function of Cs under the condition that $\mathrm{Cs}+\mathrm{Cp}(1041 \mathrm{pF})$ was kept constant. The arrows indicate the SNRs corresponding to the same condition as shown in Fig. 4 (a-c). Solid lines indicate the fitting results.

Fig. 6 Envelopes of the transimission siganl (excitation pulse) with the pulse length of $250 \mu$ s and VDD for the class-D amplifier 20V under different configurations. The config.1: diodes D1,D3, D6 and D7 were bypassed by wirings and the low side MOSFET Q3 was kept in the on state after the transimission. The config. 2: the low side MOSFET Q3 was kept in the on state after the transimission. The config. 3: the low side MOSFET Q3 was set at the off state after the transimission.

Fig. 7 Maxiumum voltage (kVpp) across the probe coil plotted as a function of VDD (V) for the proposed class-D amplifier with $(\triangle)$ the config.1 or $(O)$ the config.3. For comparison with a $50 \Omega$ transmitter system, the voltage across the coil was also plotted as a function of the output voltage (Vpp) from a $50 \Omega$ power amplifier $(\square)$. 
Fig. 8 A nitrogen-14 NQR FID signal (1.37MHz) from imidazole (30g) obtained with the proposed transceiver circuit. The excitation pulse and the $\mathrm{Q}$ factors of the resonant circuit are indicated in the upper figure. The signal accumulation was set as 300 times so that the signal during the low Q state was apparent. (a) The FID signal obtained under the low Q state and the high Q state by delaying the Q switch timing of $t_{\text {delay }}=365 \mu \mathrm{s}$. (b) The FID signal obtained under the high Q state with $t_{\text {delay }}=50 \mu \mathrm{s}$ in order to show the dead time of the receiver circuit. 
Table

Table 1. Specifications for low noise op-amps for the TIA and $50 \Omega$ wideband LNAs provided by the manufacturer's datasheets.

\begin{tabular}{c|c|c|c|c}
\hline $\begin{array}{c}\text { OP amp for } \\
\text { TIA }\end{array}$ & GBW $(\mathrm{GHz})$ & $\begin{array}{l}\text { Voltage imput } \\
\text { noise }(\mathrm{nV} / \sqrt{\mathrm{Hz}})\end{array}$ & $\begin{array}{l}\text { Current imput } \\
\text { noise }(\mathrm{pA} / \sqrt{\mathrm{Hz}})\end{array}$ & NF \\
\hline CLC1001 & 2.1 & 0.6 & 4.2 & \\
LT6200-10 & 1.6 & $\sim 1$ & 3.5 & $\mathrm{NF}$ \\
\hline $50 \Omega$ LNA & $\begin{array}{c}\text { Frequency } \\
\text { range }(\mathrm{MHz})\end{array}$ & $\begin{array}{l}\text { Voltage imput } \\
\text { noise }(\mathrm{nV} / \sqrt{\mathrm{Hz}})\end{array}$ & $\begin{array}{l}\text { Current imput } \\
\text { noise }(\mathrm{pA} / \sqrt{\mathrm{Hz}})\end{array}$ & \\
\hline amp1 & $0.1 \sim 500$ & & & $3^{\mathrm{a}}$ \\
amp2 & $1 \sim 500$ & & 5 & $1^{\mathrm{b}}$ \\
amp3 & $0.001 \sim 100$ & 0.25 & $5.6^{\mathrm{c}}$ \\
\hline
\end{tabular}

Table.2 The values obtained from the fitting of the spectral noise densities for two TIAs. The total simulated noise density $V_{\text {total }}$ and its contribution from the resistor thermal noise $V_{\mathrm{r}}$ at the resonant frequency and at the temperature of $300 \mathrm{~K}$ are also summarized.

\begin{tabular}{|c|c|c|c|c|c|c|c|}
\hline \multirow{2}{*}{ CLC1001 } & \multicolumn{3}{|c|}{$\mathrm{Cs}(\mathrm{pF}) \mathrm{Cp}(\mathrm{pF}) \mathrm{R}(\Omega)$} & \multirow{2}{*}{\begin{tabular}{|c|}
$\mathrm{Cs} "(\mathrm{pF})$ \\
844
\end{tabular}} & \multicolumn{3}{|c|}{$V_{\text {total }}(\mathrm{nV} / \sqrt{\mathrm{Hz}}) V_{\mathrm{r}}(\mathrm{nV} / \sqrt{\mathrm{Hz}}) \mathrm{NF}$} \\
\hline & 20 & 1021 & 1.5 & & 11 & 4.2 & 8.0 \\
\hline & 94 & 947 & 1.6 & 845 & 23 & 20 & 1.3 \\
\hline & 141 & 900 & 1.6 & 844 & 34 & 30 & 1.2 \\
\hline & 407 & 634 & 1.8 & 843 & 122 & 76 & 4.1 \\
\hline & 1041 & 0 & 3.1 & 834 & 334 & 126 & 8.5 \\
\hline \multirow[t]{5}{*}{ LT6200-10 } & 20 & 1021 & 1.5 & 847 & 9.1 & 4.4 & 6.3 \\
\hline & 94 & 947 & 1.5 & 846 & 25 & 20 & 1.6 \\
\hline & 141 & 900 & 1.5 & 845 & 40 & 30 & 2.3 \\
\hline & 540 & 501 & 1.7 & 847 & 285 & 97 & 9.3 \\
\hline & 1041 & 0 & 2.2 & 849 & 660 & 137 & 14 \\
\hline
\end{tabular}


(a)

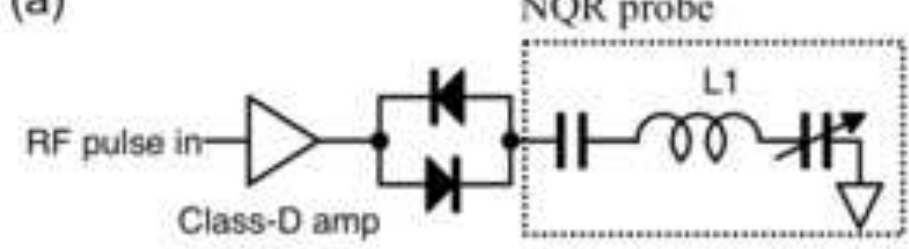

(b)



(c)






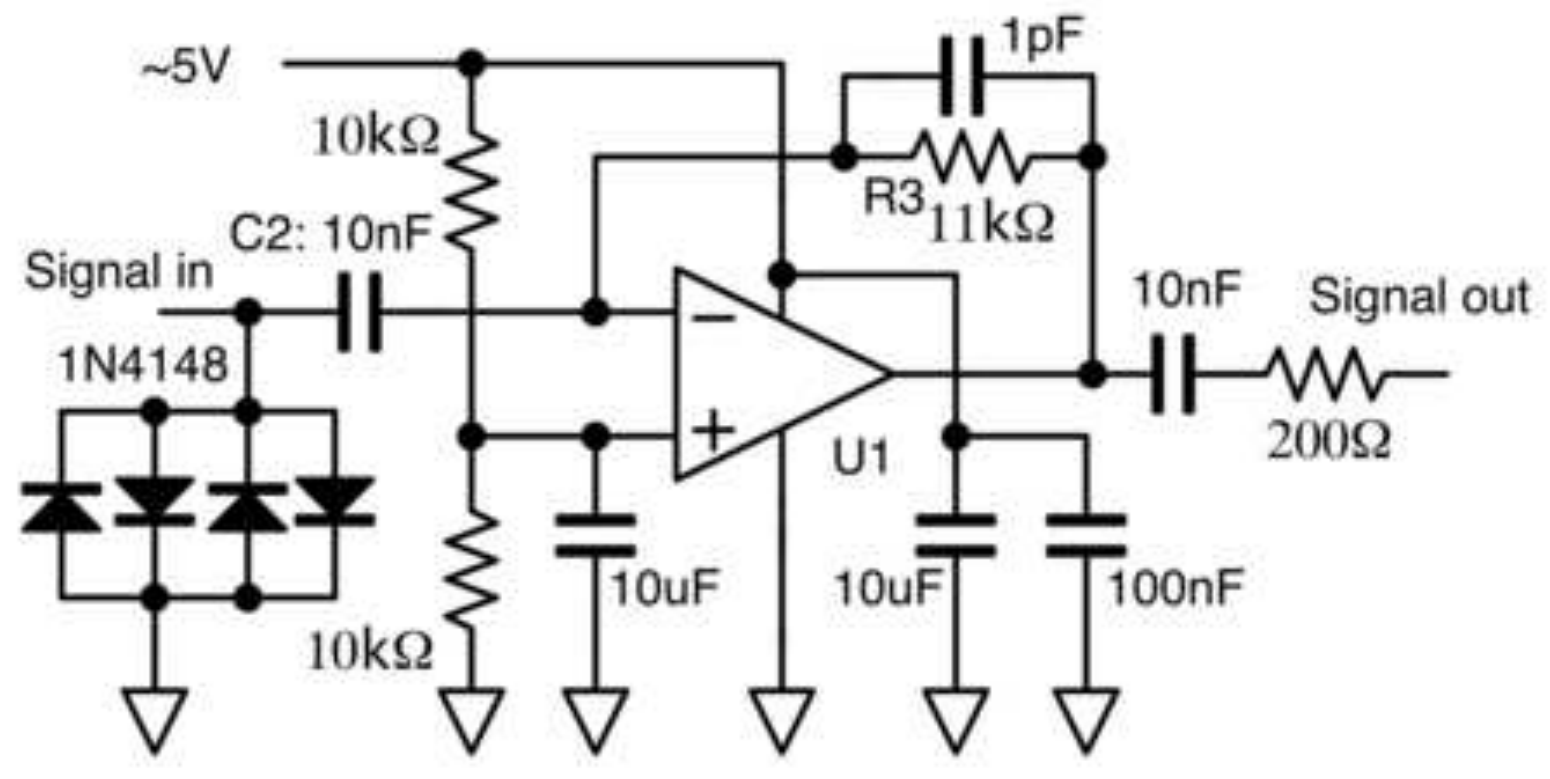







(a)

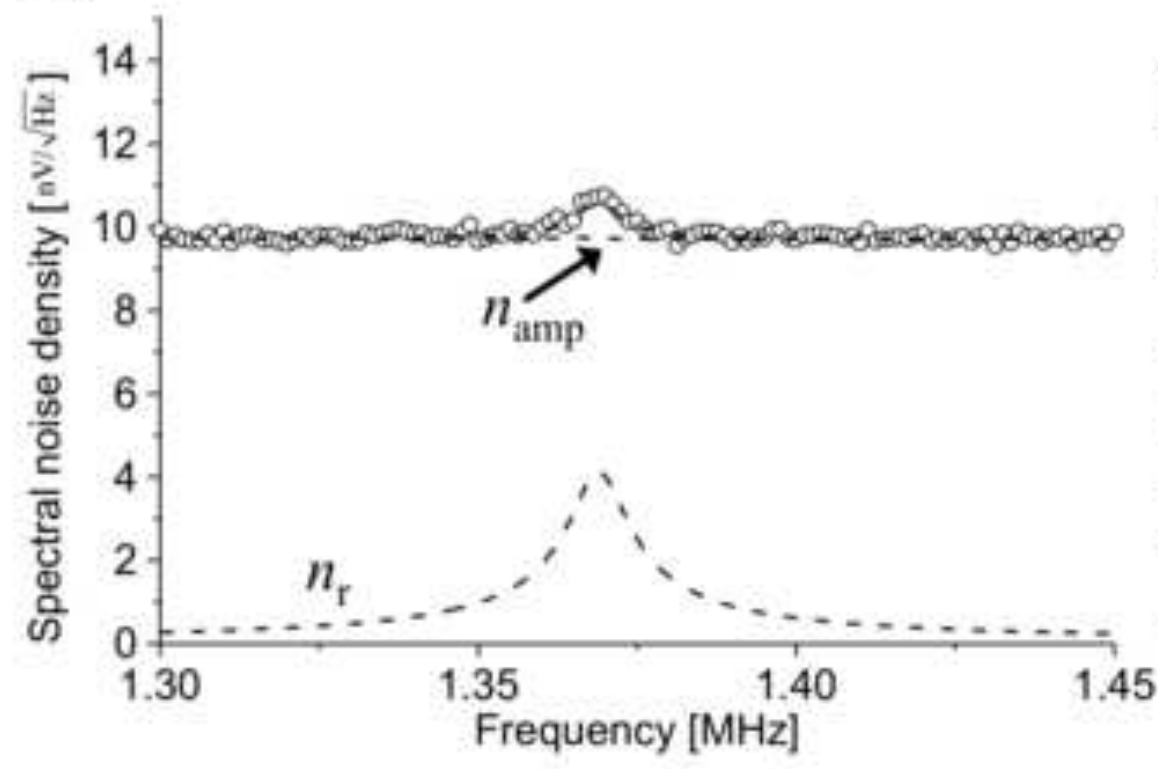

(c)

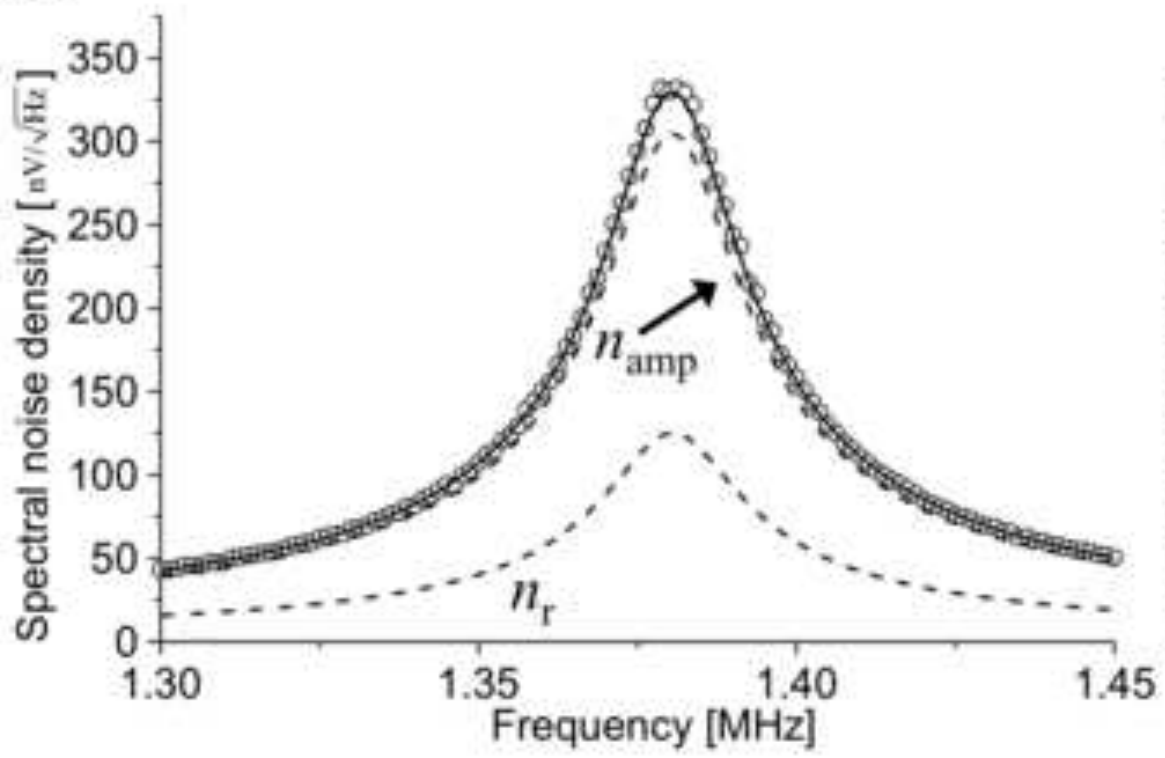

(b)

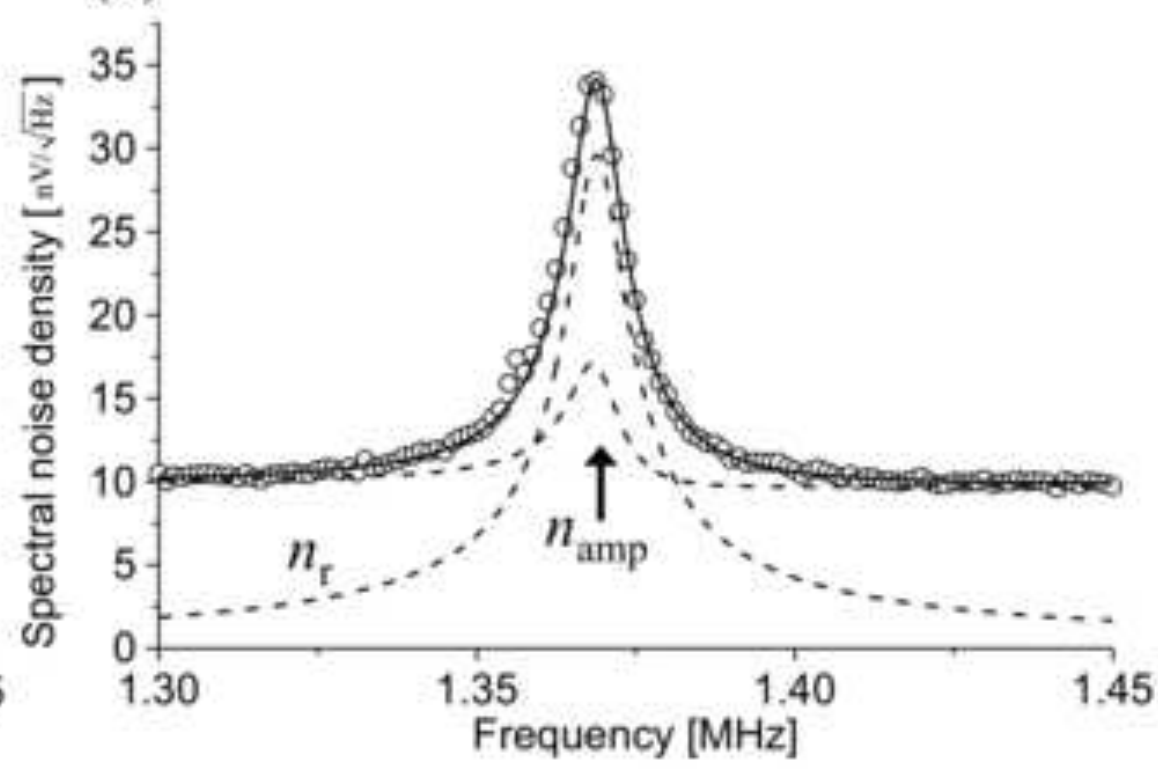

(d)

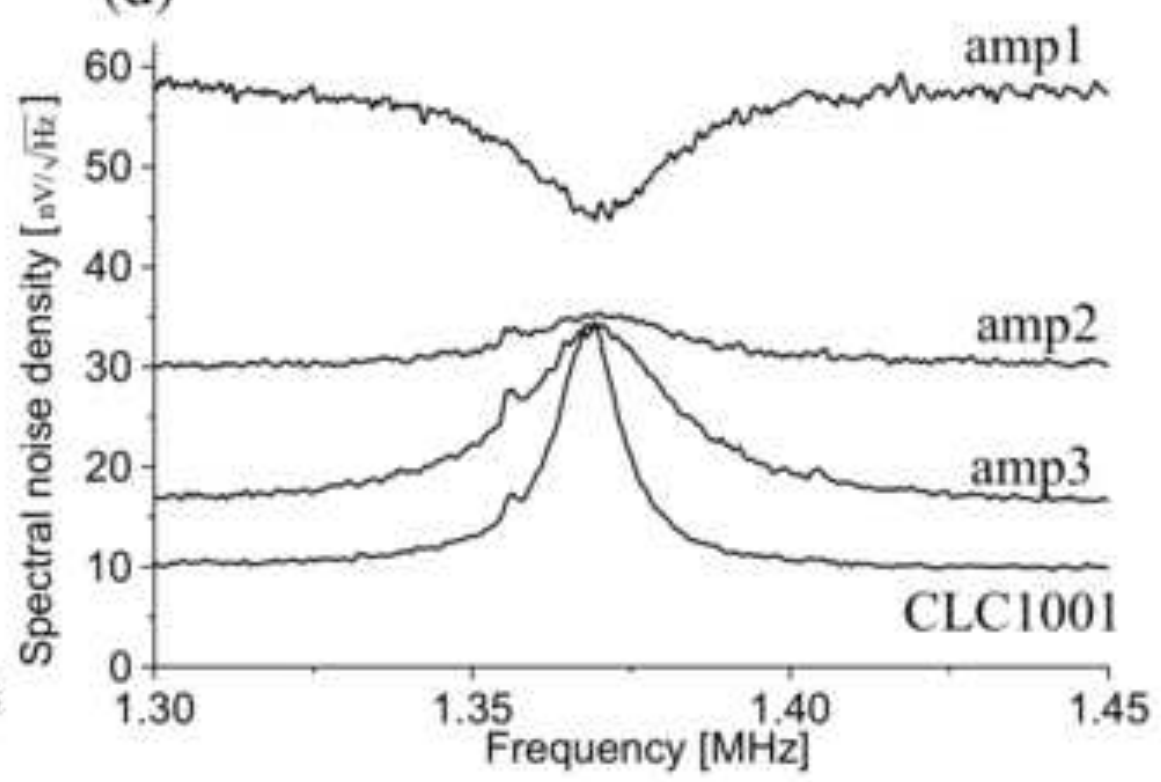




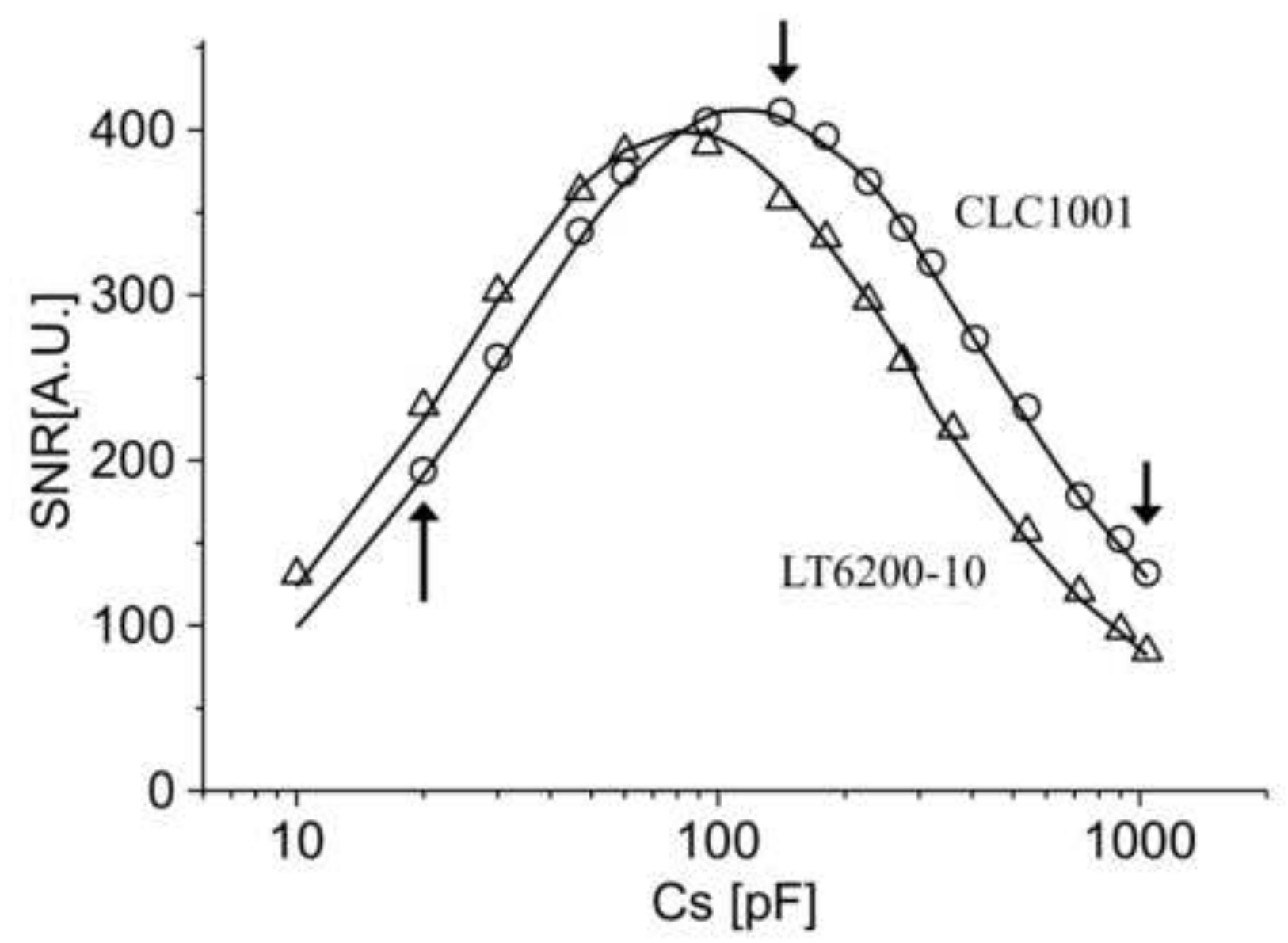




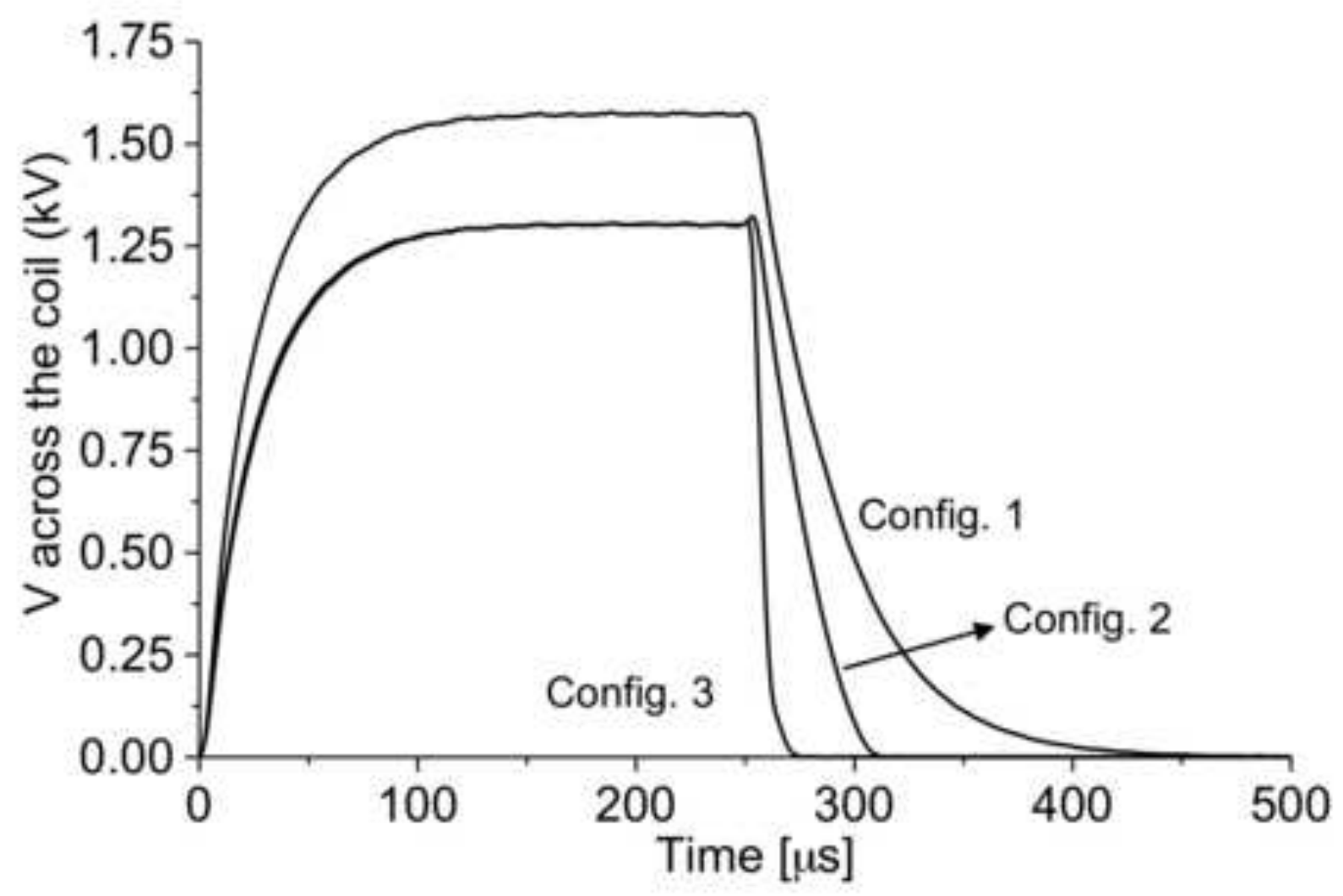




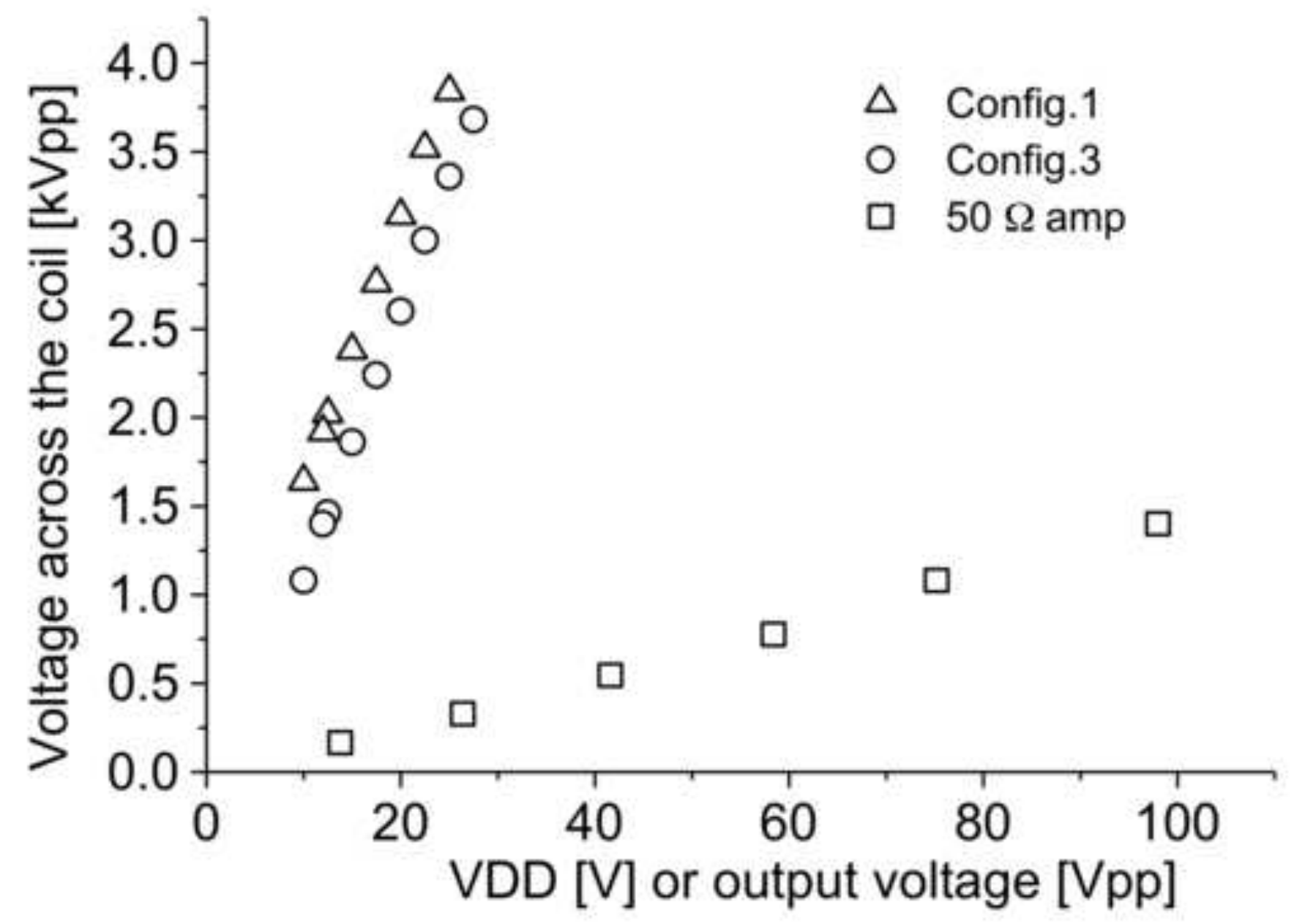


(a)

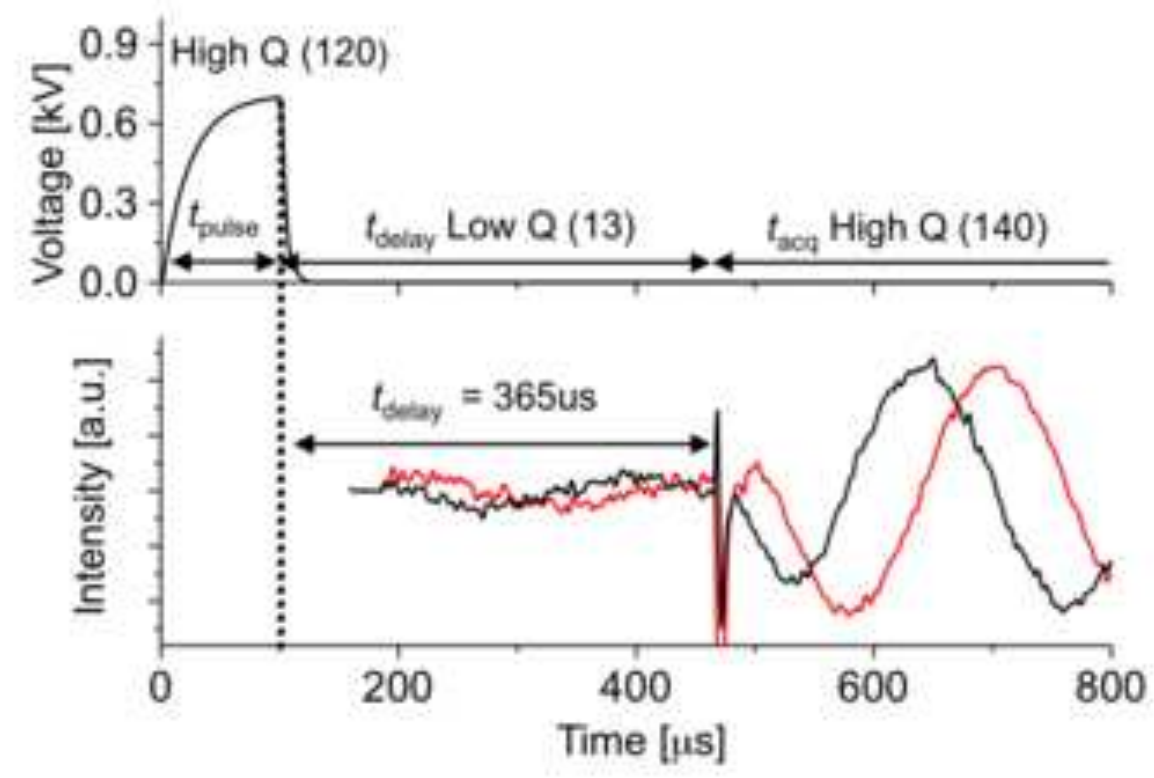

(b)

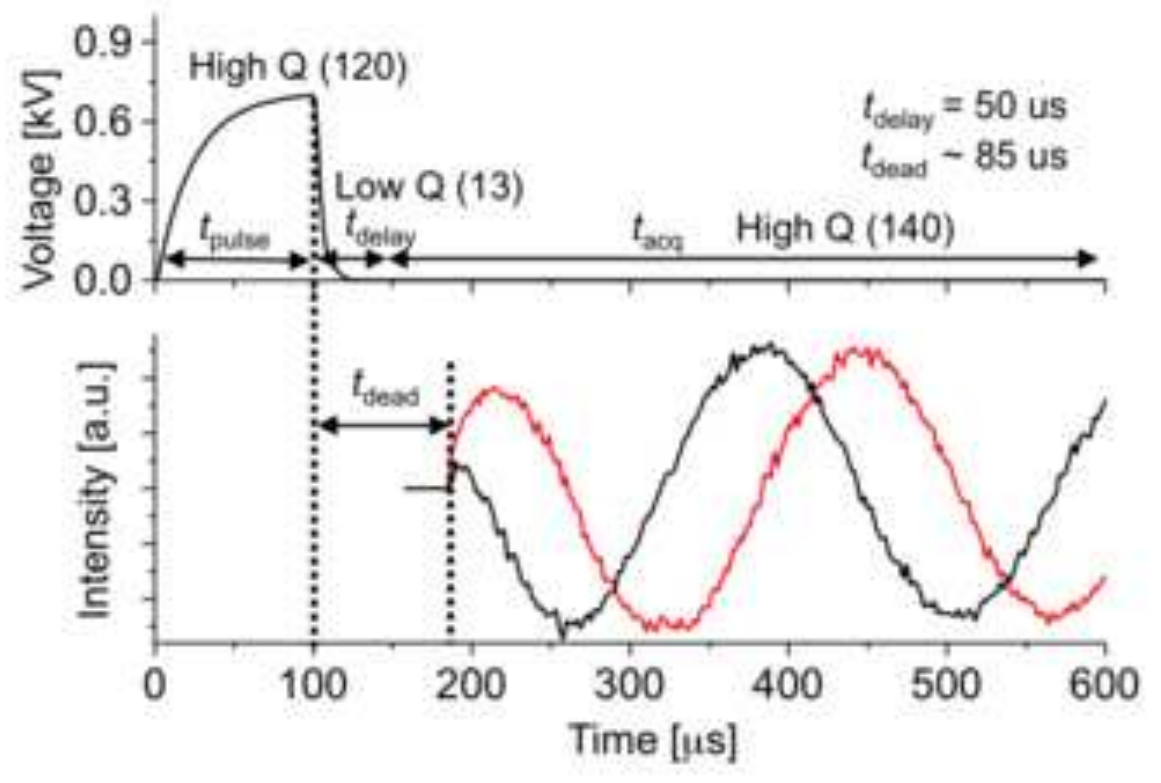




\section{Transmission}

NQR probe


Transmission/Reception

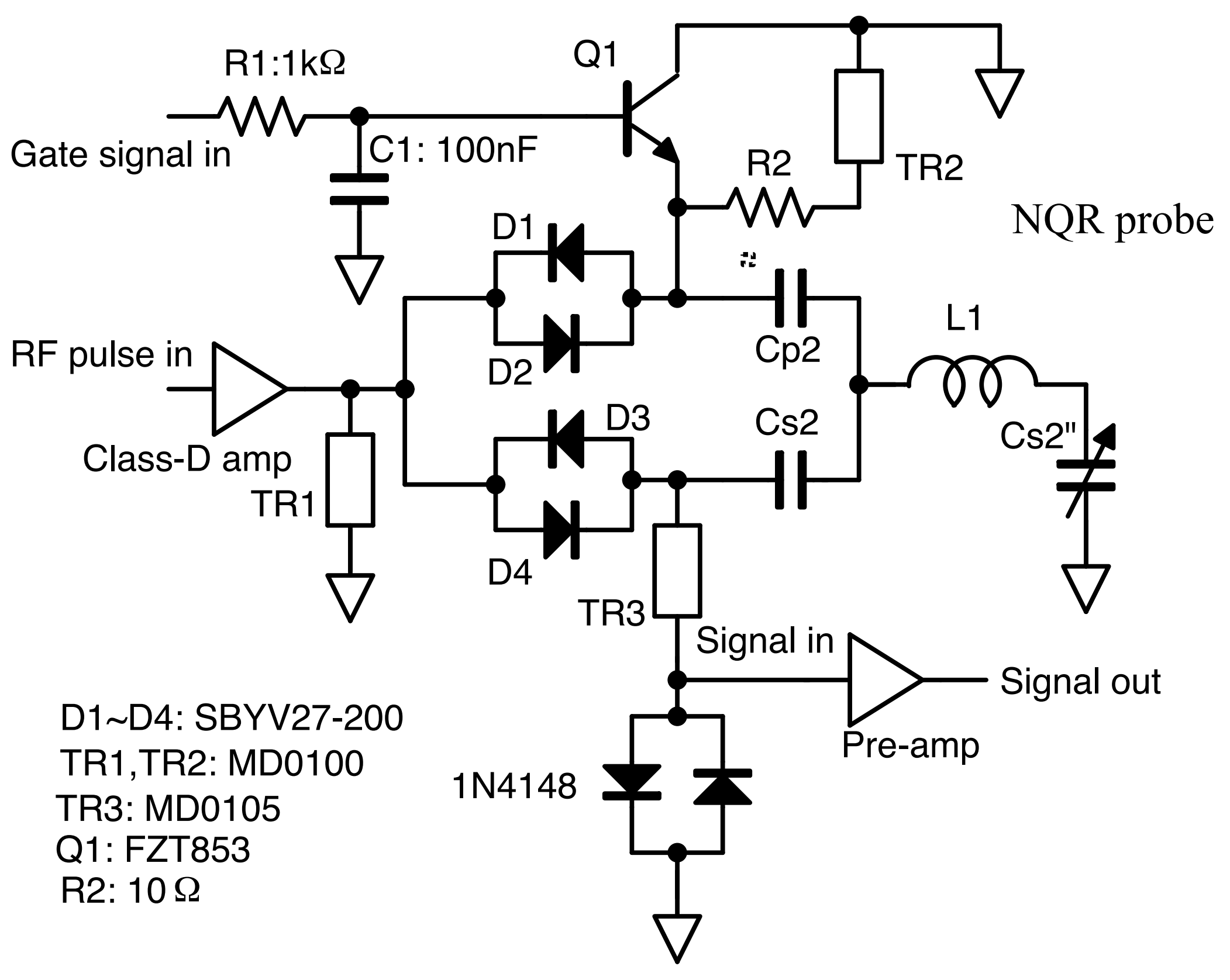

